\title{
Distributed adaptive consensus output regulation of network-connected heterogeneous unknown linear systems on directed graphs \\ Dol:
}

10.1109/TAC.2016.2628643

\section{Document Version \\ Accepted author manuscript}

Link to publication record in Manchester Research Explorer

Citation for published version (APA):

Ding, Z. (2016). Distributed adaptive consensus output regulation of network-connected heterogeneous unknown linear systems on directed graphs. IEEE Transactions on Automatic Control, [IEEE-TAC 16-1329].

https://doi.org/10.1109/TAC.2016.2628643

\section{Published in:}

IEEE Transactions on Automatic Control

\section{Citing this paper}

Please note that where the full-text provided on Manchester Research Explorer is the Author Accepted Manuscript or Proof version this may differ from the final Published version. If citing, it is advised that you check and use the publisher's definitive version.

\section{General rights}

Copyright and moral rights for the publications made accessible in the Research Explorer are retained by the authors and/or other copyright owners and it is a condition of accessing publications that users recognise and abide by the legal requirements associated with these rights.

\section{Takedown policy}

If you believe that this document breaches copyright please refer to the University of Manchester's Takedown Procedures [http://man.ac.uk/04Y6Bo] or contact uml.scholarlycommunications@manchester.ac.uk providing relevant details, so we can investigate your claim.

\section{OPEN ACCESS}




\section{Distributed Adaptive Consensus Output Regulation of Network-Connected Heterogeneous Unknown Linear Systems on Directed Graphs}

Zhengtao Ding

\begin{abstract}
This technical note deals with output regulation of network connected linear systems under directed connection graphs. The subsystem dynamics are unknown and heterogeneous. The desired output signal, generated by a linear exosystem, is only available to some subsystems, and the others will estimate the exosystem state through the network connections for obtaining regulation errors. The exosystem is parametrised in a specific form such that it has a skew-symmetric system matrix, whose property is further explored in the estimation of exosystem state, and in the estimation of the desired feed-forward control inputs for output regulation. The unknown subsystem parameters are dealt with by the adaptive laws, driven by the estimated regulation errors. The proposed adaptive control strategy is fully distributed, in the sense that the control design only uses the information in the connected neighbourhood, without reference to the eigenstructure of the associated Laplacian matrix, as long as the connection graph is strongly connected.
\end{abstract}

Index Terms-Consensus control, Adaptive control, Output regulation, Multi-agent systems

\section{INTRODUCTION}

Control of network-connected dynamic systems has attracted significant amount of attention in control research due to its applications in areas such as multi-agent systems and formation control etc. Consensus control of network-connected systems deals with the control design to ensure that all the subsystems achieve the same control objective, such as common output or state values. A significant progress in consensus control design is the use of the eigen-structure of the Laplacian matrix associated with the connection graph [1], [2], [3], [4], in particular, the least real part of the eigenvalues, or the connectivity of the graph. There are more diverse results relating to consensus control in recent years, including adaptive control, output regulation and synchronisation, and semiglobal stability (see [5], [6], [7], for example). For adaptive control of network connected systems when the connection graph is undirected, the symmetry of the Laplacian matrix is exploited in the design of adaptive laws [8], [9], [10]. Adaptive consensus control with directed connection graphs is more challenging. One way to alleviate the problem is to use robust adaptive laws at the cost of asymptotic convergence [11], [12]. Adaptive consensus tracking under directed connection graphs is addressed in [13] for formation control via state feedback. Adaptive laws are also proposed to estimate network connectivity, aiming at a fully distributed control, without using the knowledge of eigenvalues of Laplacian matrices [14]. In particular, a recent result [15] can deal with parametric uncertainty of the subsystems with a fully distributed control. But the subsystems must have identical system dynamics with relative degree one, and the regulated output must be a constant.

We consider output regulation of network-connect systems of general SISO unknown linear subsystems on directed connection graphs via output feedback. The subsystems are allowed to have different dynamics, and only some of them have access to the desired output signal. The regulation errors are obtained through the estimation of the exosystem state when the subsystems have no access to the desired output. This estimation is based on a specific parametrisation of the exosystem with a skew-symmetric system

Zhengtao Ding is with Control Systems Centre, School of Electrical and Electronic Engineering, University of Manchester, Sackville Street Building, Manchester M13 9PL, UK. Email:zhengtao.ding@manchester.ac.uk matrix, using the relative output signals from the neighbouring subsystems. The lack of the direct measurement of output regulation errors makes the internal model design for the desired feedforward inputs difficult. To tackle this problem, we integrate the estimation of the desired feedforward inputs with other adaptive laws, driven by the estimated output regulation errors, and by further exploiting the skew-symmetry of the exosystem matrix. The use of the estimated output regulation errors for the design of adaptive laws, including the estimation for feedforward control inputs, make the adaptive laws fully distributed, without using the eigenstructure of the associated Laplacian matrices. Adaptive backstepping is used for the control design of subsystems with higher relative degrees than one. The stability analysis is carried out to establish the boundedness of all the variables of the closed-loop adaptive control systems and the asymptotic convergences to zero of the output regulation errors in all the subsystems.

\section{Problem Formulation}

In this technical note, we consider a set of $N$ SISO unknown linear subsystems, of which the subsystems are described by

$$
\begin{aligned}
\dot{x}_{i} & =A_{c i} x_{i}+\phi_{i} y_{i}+b_{i} u_{i}, \\
y_{i} & =C_{i} x_{i},
\end{aligned}
$$

with $\phi_{i}, b_{i}, C_{i}^{T} \in \mathbb{R}^{n_{i}}$ and

$$
\begin{aligned}
& A_{c i}=\left[\begin{array}{cc}
0_{\left(n_{i}-1\right) \times 1} & I_{n_{i}-1} \\
0 & 0_{1 \times\left(n_{i}-1\right)}
\end{array}\right], \\
& b_{i}=\left[\begin{array}{llllll}
0 & \ldots & 0 & b_{i, \rho} & \ldots & b_{i, n_{i}}
\end{array}\right]^{T}, \\
& C_{i}^{T}=\left[\begin{array}{llll}
1 & 0 & \ldots & 0
\end{array}\right]
\end{aligned}
$$

for $i=1, \ldots N$, where $x_{i} \in \mathbb{R}^{n_{i}}$ is the state vector, with $n_{i}$ being known positive constant integers, denoting the order of the subsystems, $y_{i}, u_{i} \in \mathbb{R}$ are the output and input respectively of the $i$ th subsystem, and $\phi_{i}, b_{i} \in \mathbb{R}^{n_{i}}$ are vectors of unknown parameters, with the high-frequency gains $b_{i, \rho} \neq 0$, which implies the relative degree of the subsystem is $\rho$.

The connections between the subsystems are specified by a directed graph $\mathcal{G}$ that consists of a set of vertices denoted by $\mathcal{V}$ and a set of edges denoted by $\mathcal{E}$. A vertex represents a subsystem, and each edge represents a connection. Associated with the graph, its adjacency matrix $A$ with elements $a_{i j}$ denotes the connections such that $a_{i j}=1$ if there is a path from subsystem $j$ to subsystem $i$, and $a_{i j}=0$ otherwise. We define the Laplacian matrix $L$ is a normal way as $l_{i i}=\sum_{j=1}^{N} a_{i j}$ and $l_{i j}=-a_{i j}$ when $i \neq j$.

We define the output regulation errors as

$$
e_{i}=y_{i}-h^{T} w_{0}
$$

with $h \in \mathbb{R}^{n_{s}}$ for $i=1, \ldots, N$, where $w_{0}$ is generated by an exosystem

$$
\dot{w}_{0}=S w_{0}
$$

where $S \in \mathbb{R}^{n_{s} \times n_{s}}$ is a constant matrix.

Not all the subsystems have the access to the function value of $h^{T} w_{0}$. We use a diagonal matrix $\Delta$ to denote the access to $h^{T} w_{0}$ in the way that if $\delta_{i}=1$, the $i$ th subsystem has access to the value of $h^{T} w_{0}$ for the control design, and $\delta_{i}=0$ otherwise. At least one subsystem has the access. The subsystems which do not have access to the desired output $h^{T} w_{0}$ rely on the network connections to achieve the consensus output regulation.

The adaptive consensus output regulation problem considered in this technical note is to design an adaptive control strategy using the relative output information $y_{i}-y_{j}, i \neq j$, provided by the network connection to each subsystem to ensure the convergence to 
zero of output regulation errors $e_{i}$ for $i=1, \ldots, N$ under any initial condition of the system in the state space, i.e., the convergence of the subsystem outputs $y_{i}$ to the common function $h^{T} w_{0}$.

We make several assumptions about the dynamics of the subsystems, the exosystem and the connections between the subsystems.

Assumption 1. The invariant zeros of $\left\{A_{c i}, b_{i}, C_{i}\right\}$ are stable, for $i=1, \ldots, N$, and all the subsystems have the known signs of the high-frequency gains.

Assumption 2. The eigenvalues of $S$ are distinct and on the imaginary axis.

Assumption 3. The network graph is strongly connected.

Note that the system shown in (1) is in the observer canonical form, and any controllable and observable systems can be transformed to such a structure. Here we assume that the subsystems are with the known orders and known signs of high-frequency gains, and all the other parameters are completely unknown.

\section{PRELIMINARIES}

Several preliminary results are needed for proposing the adaptive control design later.

We introduce filtered transformation with the filter for the subsystem $i$, for $i=1, \ldots, N$,

$$
\begin{aligned}
\dot{\xi}_{i, 1} & =-\lambda_{1} \xi_{i, 1}+\xi_{i, r} \\
& \cdots \\
\dot{\xi}_{i, \rho-1} & =-\lambda_{\rho-1} \xi_{i, \rho-1}+u_{i},
\end{aligned}
$$

where $\lambda_{j}>0$ for $j=1, \ldots, \rho-1$ are the design parameters, and the filtered transformation

$$
\bar{z}_{i}=x_{i}-\left[\bar{d}_{i, 1} \ldots \bar{d}_{i, \rho-1}\right] \xi_{i},
$$

where $\xi_{i}=\left[\xi_{i, 1} \ldots \xi_{i, \rho-1}\right]^{T}, \bar{d}_{i, j} \in \mathbb{R}^{n_{i}}$ for $j=1, \ldots, \rho-1$ and they are generated recursively by $\bar{d}_{i, \rho-1}=b_{i}$ and $\bar{d}_{i, j}=$ $\left(A_{c i}+\lambda_{j+1} I\right) \bar{d}_{i, j+1}$ for $j=\rho-2, \ldots, 1$. The system (1) is then transformed to

$$
\begin{aligned}
\dot{\bar{z}}_{i} & =A_{c i} \bar{z}_{i}+\phi_{i} y_{i}+d_{i} \xi_{i, 1} \\
y_{i} & =C \bar{z}_{i},
\end{aligned}
$$

where $d_{i}=\left(A_{c i}+\lambda_{1} I\right) \bar{d}_{i, 1}$. It can be shown that $d_{i, 1}=b_{i, \rho}$ and

$$
\sum_{j=1}^{n_{i}} d_{i, j} s^{n_{i}-j}=\prod_{j=1}^{\rho-1}\left(s+\lambda_{j}\right) \sum_{j=\rho}^{n_{i}} b_{i, j} s^{n_{i}-j} .
$$

With $\xi_{i, 1}$ as the input, the system (6) is with relative degree one and minimum phase. We introduce another state transformation to extract the internal dynamics of (6) with $z_{i} \in \mathbb{R}^{n_{i}-1}$ given by

$$
z_{i}=\bar{z}_{: n_{i}}-\frac{d_{i, 2: n_{i}}}{d_{i, 1}} y_{i}
$$

where $(\cdot)_{2: n_{i}}$ refers to the vector or matrix formed by the 2 nd row to the $n_{i}$ th row. With the coordinates $\left(z_{i}, y_{i}\right),(6)$ is rewritten as

$$
\begin{aligned}
& \dot{z}_{i}=D_{i} z_{i}+\psi_{i} y_{i} \\
& \dot{y}_{i}=z_{i, 1}+\psi_{i, y} y_{i}+b_{i, \rho} \xi_{i, 1},
\end{aligned}
$$

where $D_{i}$ is the left companion matrix of $d_{i}$ given by

$$
D_{i}=\left[\begin{array}{cccc}
-d_{i, 2} / d_{i, 1} & 1 & \ldots & 0 \\
-d_{i, 3} / d_{i, 1} & 0 & \ddots & 0 \\
\vdots & \vdots & \vdots & \vdots \\
-d_{i, n_{i}-1} / d_{i, 1} & 0 & \ldots & 1 \\
-d_{i, n_{i}} / d_{i, 1} & 0 & \ldots & 0
\end{array}\right]
$$

and

$$
\begin{aligned}
\psi_{i} & =D_{i} \frac{d_{i, 2: n_{i}}}{d_{i 1}}+\phi_{i, 2: n_{i}}-\frac{d_{i, 2: n_{i}}}{d_{i, 1}} \phi_{i, 1} \\
\psi_{i, y} & =\frac{d_{i, 2}}{d_{i, 1}}+\phi_{i, 1} .
\end{aligned}
$$

For the exosystem, since its system matrix has distinct eigenvalues on the imaginary axis, we transform the exosystem in a special form with a known constant output matrix.

Lemma 3.1 Consider a linear system

$$
\dot{z}=F z, \text { with } z(0)=z_{\alpha}, \quad y=g^{T} z
$$

where $z \in \mathbb{R}^{n_{s}}$ is the state vector, with $n_{s}$ an known positive constant integers, $y \in \mathbb{R}$ is the output, $g \in \mathbb{R}^{n_{s}}$ is an unknown vector, $z_{\alpha} \in$ $\mathbb{R}^{n_{s}}$ is an unknown initial state, and $F \in \mathbb{R}^{n_{s} \times n_{s}}$ has known distinct eigenvalues on the imaginary axis. There exists a state transformation with $w=T z$, such that the system dynamics are described by

$$
\dot{w}=S w, \text { with } w(0)=w_{\alpha}, \quad y=h^{T} w
$$

where $F \in \mathbb{R}^{n_{s} \times n_{s}}$ is a known skew-symmetric block diagonal matrix, and $h \in \mathbb{R}^{n_{s}}$ is a known constant vector, $w_{\alpha} \in \mathbb{R}^{n_{s}}$ is an unknown initial state.

Proof. The system order $n_{s}$ can be an even or odd positive integer. For an odd $n_{s}$, the system has a single eigenvalue at 0 , indicating that the output contains a constant bias. For the convenience of presentation, we show the proof for the even order.

Since the eigenvalues of $F$ are distinct and on the imaginary axis, there exists a non-singular $T \in \mathbb{R}^{n_{s} \times n_{s}}$ such that $T F T^{-1}=S$, with $S=\operatorname{diag}\left\{S_{1}, \ldots, S_{n_{s} / 2}\right\}$, of which $S_{i}=\left[\begin{array}{cc}0 & \omega_{i} \\ -\omega_{i} & 0\end{array}\right]$ with $\omega_{i}$ being positive real numbers, indicating the frequencies of the output signal. Let $\bar{g}=T^{-T} g$ and $\bar{z}_{\alpha}=T z_{\alpha}$. With $e^{S_{i} t}=\left[\begin{array}{cc}\cos \omega_{i} t & \sin \omega_{i} t \\ -\sin \omega_{i} t & \cos \omega_{i} t\end{array}\right]$, the system output is given by

$$
\begin{aligned}
y= & \sum_{i=1}^{n_{s} / 2}\left(\left(\bar{g}_{2 i-1} \bar{z}_{\alpha, 2 i-1}+\bar{g}_{2 i} \bar{z}_{\alpha, 2 i}\right) \cos \omega_{i} t\right. \\
& \left.+\left(\bar{g}_{2 i-1} \bar{z}_{\alpha, 2 i}-\bar{g}_{2 i} \bar{z}_{\alpha, 2 i-1}\right) \sin \omega_{i} t\right) .
\end{aligned}
$$

Considering the output $y$ with the state $w$, we obtain the components of $w_{\alpha}$ as, for $i=1, \ldots, n_{s} / 2$,

$$
\begin{aligned}
w_{\alpha, 2 i-1} & =\bar{g}_{2 i-1} \bar{z}_{\alpha, 2 i-1}+\bar{g}_{2 i} \bar{z}_{\alpha, 2 i}, \\
w_{\alpha, 2 i} & =\bar{g}_{2 i-1} \bar{z}_{\alpha, 2 i}-\bar{g}_{2 i} \bar{z}_{\alpha, 2 i-1},
\end{aligned}
$$

and $h=[1,0,1,0, \ldots, 1,0]^{T}$.

Based on Lemma 3.1, we can assume that the exosystem in (3) has a known constant output vector $h$ and a known skew-symmetric system matrix $S$. Before we introduce the control design, we need a result on the Laplacian matrix.

Lemma 3.2: [16], [15] If the adjacency matrix $A$ whose corresponding connection graph is strongly connected, and the nonnegative diagonal matrix $\Delta$ has at least one positive diagonal element, there exist a positive diagonal matrix $G$ with $G=\operatorname{diag}\left\{g_{1}, \ldots, g_{N}\right\}$ such that

$$
G(L+\Delta)+(L+\Delta)^{T} G \geq r_{0} I
$$

for some positive real number $r_{0}$, where $L$ is the Laplacian matrix, corresponding to $A$.

Let us denote $e=\left[e_{1}, e_{2}, \ldots, e_{N}\right]^{T}$, and the consensus regulation error $\zeta=Q e$ where $Q=L+\Delta$, with $\Delta=\operatorname{diag}\left\{\delta_{1}, \ldots, \delta_{N}\right\}$. It is worth noting that $\zeta=Q e$ implies that

$$
\zeta_{i}=\sum_{j=1}^{N} q_{i j} e_{j}=\sum_{j=1}^{N} a_{i j}\left(y_{i}-y_{j}\right)+\delta_{i}\left(y_{i}-h^{T} w_{0}\right)
$$


for $i=1, \ldots, N$, where $q_{i j}$ are the elements of $Q$. Clearly, $\zeta_{i}$ is available to the control design for the $i$ th subsystem.

\section{Control Design, $\rho=1$}

For $\rho=1$, we have $\xi_{i, 1}=u_{i}$, and from (9), the output dynamics of the subsystem $i$ is

$$
\dot{y}_{i}=z_{i, 1}+\psi_{i, y} y_{i}+b_{i, \rho} u_{i} .
$$

Let

$$
\begin{aligned}
z_{i 0} & =F_{i} w_{0} \\
\eta_{i} & =\frac{1}{b_{i}}\left(h^{T} S w_{0}-\bar{z}_{i, 1}-\psi_{i, y} y_{0}\right),
\end{aligned}
$$

where $F_{i} \in \mathbb{R}^{\left(n_{i}-1\right) \times n_{s}}$ is a solution of

$$
F_{i} S-D_{i} F_{i}=\psi_{i} h^{T} .
$$

Note the solution of $F_{i}$ is guaranteed by the fact that $S$ and $D_{i}$ have exclusive eigenvalues. The subsystem dynamics can be rewritten as

$$
\begin{aligned}
& \dot{\tilde{z}}_{i}=D_{i} \tilde{z}_{i}+\psi_{i} e_{i} \\
& \dot{e}_{i}=\tilde{z}_{i, 1}+\psi_{i, y} e_{i}+b_{i, \rho}\left(u_{i}-\eta_{i}\right)
\end{aligned}
$$

where $\tilde{z}_{i}=z_{i}-z_{i 0}$

The regulation error $e_{i}$ is available for control design only if $\delta_{i}=$ 1 . In case of $\delta_{i}=0$, we propose to use an estimate of the regulation error given by

$$
\epsilon_{i}=y_{i}-h^{T} \hat{w}_{0 i}
$$

for control design, where $\hat{w}_{0 i}$ is an estimate of $w_{0}$ in the subsystem $i$. We can unify the notation by defining

$$
\begin{aligned}
\epsilon_{i} & :=e_{i}+\left(1-\delta_{i}\right)\left(y_{0}-h^{T} \hat{w}_{0 i}\right) \\
& =e_{i}+\left(1-\delta_{i}\right) h^{T} \tilde{w}_{0 i}
\end{aligned}
$$

where $\tilde{w}_{0 i}=w_{0}-\hat{w}_{0 i}$. From (15), the dynamics of $\epsilon_{i}$ is obtained as

$$
\dot{\epsilon}_{i}=\tilde{z}_{i, 1}+\psi_{i, y} e_{i}+b_{i, \rho}\left(u_{i}-\eta_{i}\right)+\left(1-\delta_{i}\right) h^{T} \dot{\tilde{w}}_{0 i} .
$$

Let

$$
u_{i}=\hat{\varrho}_{i} \bar{u}_{i},
$$

where $\hat{\varrho}_{i}$ is an estimate of $\varrho_{i}:=1 / b_{i, \rho}$, with the adaptive law

$$
\dot{\hat{\varrho}}_{i}=-\operatorname{sgn}\left(b_{i, \rho}\right) \epsilon_{i} \bar{u}_{i} .
$$

The dynamics of the estimated regulation error can be written as

$$
\dot{\epsilon}_{i}=\tilde{z}_{i, 1}+\psi_{i, y} e_{i}-\left(1-\delta_{i}\right) h^{T} \dot{\hat{w}}_{0 i}+h^{T} w_{i}-b_{i, \rho} \tilde{\varrho}_{i} \bar{u}_{i}+\bar{u}_{i}
$$

where $\tilde{\varrho}_{i}=\varrho_{i}-\hat{\varrho}_{i}$ and $\left(1-\delta_{i}\right) h^{T} S w_{0}-b_{i, \rho} \eta_{i}:=h^{T} w_{i}$ with $\dot{w}_{i}=S w_{i}$, by Lemma 3.1. Design the control input by setting

$$
\bar{u}_{i}=-k_{0 i} \epsilon_{i}-\hat{k}_{i} \zeta_{i}+\left(1-\delta_{i}\right) h^{T} \dot{\hat{w}}_{0 i}-h^{T} \hat{w}_{i}
$$

where $k_{0 i}$ is a positive real constant, and $\hat{k}_{i}$ is an adaptive gain. We design the adaptive laws as

$$
\begin{aligned}
\dot{\hat{w}}_{0 i} & =S \hat{w}_{0 i}-\left(1-\delta_{i}\right) \zeta_{i} h, \\
\dot{\hat{w}}_{i} & =S \hat{w}_{i}+\epsilon_{i} h, \\
\dot{\hat{k}}_{i} & =\epsilon_{i} \zeta_{i} .
\end{aligned}
$$

The resultant dynamics of $\epsilon_{i}$ is obtained as

$$
\dot{\epsilon}_{i}=\tilde{z}_{i, 1}+\psi_{i, y} e_{i}-k_{0 i} \epsilon_{i}-\hat{k}_{i} \zeta_{i}+h^{T} \tilde{w}_{i}-b_{i, \rho} \tilde{\varrho}_{i} \bar{u}_{i}
$$

where $\tilde{w}_{i}=w_{i}-\hat{w}_{i}$.
Theorem 4.1 For the network connected heterogeneous unknown linear subsystems (1) with relative degree $\rho=1$, the control input specified in (19) and (22) together with the adaptive laws in (23), (24) and (25) solve the consensus output tracking problem under Assumptions 1, 2 and 3 , in the sense that the regulation error $e_{i}$ converges to zero asymptotically for $i=1, \ldots, N$.

Proof. Let

$$
V_{\epsilon 1}=\frac{1}{2} \sum_{i=1}^{N} g_{i}\left(\epsilon_{i}^{2}+\left|b_{i, \rho}\right| \tilde{\varrho}_{i}^{2}+k \tilde{w}_{0 i}^{T} \tilde{w}_{0 i}+\tilde{w}_{i}^{T} \tilde{w}_{i}+\tilde{k}_{i}^{2}\right)
$$

where $\tilde{k}_{i}=k-\hat{k}_{i}$ with $k$ a positive constant to be determined later. By (21), we have

$$
\begin{aligned}
\dot{V}_{\epsilon 1}= & \sum_{i=1}^{N} g_{i}\left(-k_{0 i} \epsilon_{i}^{2}-\hat{k}_{i} \epsilon_{i} \zeta_{i}+\epsilon_{i} \tilde{z}_{i, 1}+\epsilon_{i} \psi_{i, y} e_{i}\right. \\
& +\epsilon_{i} h^{T} \tilde{w}_{i}-\epsilon_{i} b_{i, \rho} \tilde{\varrho}_{i} \bar{u}_{i}-\left|b_{i, \rho}\right| \tilde{\varrho}_{i} \dot{\hat{\varrho}}_{i} \\
& \left.+k \tilde{w}_{0 i}^{T} \dot{\tilde{w}}_{0 i}+\tilde{w}_{i}^{T} \dot{\tilde{w}}_{i}-\left(k-\hat{k}_{i}\right) \dot{\hat{k}}_{i}\right) \\
= & \sum_{i=1}^{N} g_{i}\left(-k_{0 i} \epsilon_{i}^{2}-k \epsilon_{i} \zeta_{i}+\epsilon_{i} \tilde{z}_{i, 1}+\epsilon_{i} \psi_{i, y} e_{i}\right. \\
& \left.+k \tilde{w}_{0 i}^{T} \dot{\tilde{w}}_{0 i}\right)
\end{aligned}
$$

where we have used the adaptive laws for $\hat{k}_{i}$ and $\hat{w}_{i}$ and the fact that $\tilde{w}_{i}^{T} S \tilde{w}_{i}=0$, because $S$ is skew-symmetric. Substituting $\epsilon_{i}=$ $e_{i}+\left(1-\delta_{i}\right) h^{T} \tilde{w}_{0 i}$ into (28), we have

$$
\begin{aligned}
\dot{V}_{\epsilon 1}= & \sum_{i=1}^{N} g_{i}\left(-k_{0 i} \epsilon_{i}^{2}-k e_{i} \zeta_{i}-k\left(1-\delta_{i}\right) h^{T} \tilde{w}_{0 i} \zeta_{i}\right. \\
& \left.+\epsilon_{i} \tilde{z}_{i, 1}+\epsilon_{i} \psi_{i, y} e_{i}+k \tilde{w}_{0 i}^{T} \dot{\tilde{w}}_{0 i}\right) \\
= & \sum_{i=1}^{N} g_{i}\left(-k_{0 i} \epsilon_{i}^{2}-k e_{i} \zeta_{i}+\epsilon_{i} \tilde{z}_{i, 1}+\epsilon_{i} \psi_{i, y} e_{i}\right) .
\end{aligned}
$$

Note that

$$
\sum_{i=1}^{N} g_{i} e_{i} \zeta_{i}=\frac{1}{2} e^{T}\left(G Q+Q^{T} G\right) e \geq \frac{1}{2} r_{0}\|e\|^{2}
$$

by Lemma 3.2. It follows that

$$
\begin{aligned}
\dot{V}_{\epsilon} \leq & -\frac{1}{2} k r_{0}\|e\|^{2}+\sum_{i=1}^{N} g_{i}\left(-\left(k_{0 i}-2 \kappa\right) \epsilon_{i}^{2}\right. \\
& \left.+\frac{1}{4 \kappa}\left\|\tilde{z}_{i}\right\|^{2}+\frac{\psi_{i, y}^{2}}{4 \kappa} e_{i}^{2}\right) \\
\leq & -\left(\frac{1}{2} k r_{0}-\frac{\bar{\psi}}{4 \kappa}\right)\|e\|^{2}+\frac{\bar{g}}{4 \kappa} \sum_{i=1}^{N}\left\|\tilde{z}_{i}\right\|^{2}
\end{aligned}
$$

where $\kappa$ is a positive constant that satisfies $\kappa<\frac{1}{2} \min _{i}\left\{k_{0 i}\right\}, \bar{g}:=$ $\max _{i}\left\{g_{i}\right\}$, and $\bar{\psi}:=\max _{i}\left\{\psi_{i, y}^{2}\right\}$.

Since $D_{i}$ is Hurwitz, there exists a positive definite matrix $P_{i}$ such that $P_{i} D_{i}+D_{i}^{T} P_{i}=-3 I$. Let

$$
V_{z}=\sum_{i=1}^{N} \tilde{z}_{i}^{T} P_{i} \tilde{z}_{i}
$$

From (15), it can be obtained that

$$
\begin{aligned}
\dot{V}_{z} & =-3 \sum_{i=1}^{N}\left\|\tilde{z}_{i}\right\|^{2}+2 \sum_{i=1}^{N} \tilde{z}_{i}^{T} P_{i} \psi_{i} e_{i} \\
& \leq-2 \sum_{i=1}^{N}\left\|\tilde{z}_{i}\right\|^{2}+\bar{p}_{d}\|e\|^{2}
\end{aligned}
$$


where $\bar{p}_{d}:=\max _{i}\left\{\left\|P_{i} \psi_{i}\right\|^{2}\right\}$. Let $V_{1}=V_{\epsilon 1}+\frac{\bar{g}}{4 \kappa} V_{z}$. From (30) and (32), we have

$$
\begin{aligned}
\dot{V}_{1} & \leq-\left(\frac{1}{2} k r_{0}-\frac{\bar{\psi}}{4 \kappa}-\bar{p}_{d} \frac{\bar{g}}{4 \kappa}\right)\|e\|^{2}-\sum_{i=1}^{N}\left\|\tilde{z}_{i}\right\|^{2} \\
& =-\kappa_{0}\|e\|^{2}-\frac{\bar{g}}{4 \kappa} \sum_{i=1}^{N}\left\|\tilde{z}_{i}\right\|^{2}
\end{aligned}
$$

by setting $k=\frac{2}{r_{0}}\left(\kappa_{01}+\frac{\bar{\psi}}{4 \kappa}+\bar{p}_{d} \frac{\bar{g}}{4 \kappa}\right)$ with $\kappa_{01}>0$. Hence we can conclude that all the variables in the closed-loop system are bounded, $e \in L_{2} \cap L_{\infty}, \tilde{z}_{i} \in L_{2} \cap L_{\infty}$, for $i=1, \ldots, N$, and furthermore from Babalat's lemma, that $\lim _{t \rightarrow \infty} e(t)=0$ and $\lim _{t \rightarrow \infty} \tilde{z}_{i}(t)=0$, for $i=1, \ldots, N$.

Remark 1 . For the case $\rho=1$, the only exchange of information through the network is due to the variable $\zeta_{i}$ in the control input and the adaptive laws. Other variables used in the control design are locally generated, i.e., they are generated in the subsystem.

\section{Control Design, $\rho>1$}

For the case of $\rho>1$, the subsystem dynamics can be written as

$$
\begin{aligned}
\dot{\tilde{z}}_{i} & =D_{i} \tilde{z}_{i}+\psi_{i} e_{i} \\
\dot{e}_{i} & =\tilde{z}_{i, 1}+\psi_{i, y} e_{i}+b_{i, \rho}\left(\xi_{i, 1}-\eta_{i}\right) .
\end{aligned}
$$

Comparing (33) with (15), the only difference is that $u_{i}$ is replaced by $\xi_{i, 1}$. With $\rho>1$, we cannot assign values to $\xi_{i, 1}$, but a desired value of $\xi_{i, 1}$, denoted by $\hat{\xi}_{i, 1}$, can be determined in a similar way to the control design for the case of $\rho=1$ shown in the previous section. The final control $u_{i}$ can be obtained by adaptive backstepping, following the dynamics of the filters shown in (4) in $\rho-1$ steps.

Assumption 4. All the subsystems have the known high-frequency gains.

Remark 2. This assumption is not needed for the case of $\rho=1$. It is introduced here to ease the control design in dealing with the propagation of adaptive parameters that may happen among the subsystems. It is fairly common to assume some knowledge of the high-frequency gains in adaptive control design [17], [18].

From (33) with (17), the dynamics of the estimated regulation error can be written as

$$
\begin{aligned}
\dot{\epsilon}_{i}= & \tilde{z}_{i, 1}+\psi_{i, y} e_{i}-\left(1-\delta_{i}\right) h^{T} \dot{\hat{w}}_{0 i}+h^{T} w_{i, 1}+h^{T} w_{i, 2} \\
& +b_{i, \rho} \xi_{i, 1}
\end{aligned}
$$

where $\left(1-\delta_{i}\right) h^{T} S w_{0}:=h^{T} w_{i, 1}$ with $\dot{w}_{i, 1}=S w_{i, 1}$, and $-b_{i, \rho} \eta_{i}:=$ $h^{T} w_{i, 2}$ with $\dot{w}_{i, 2}=S w_{i, 2}$, by Lemma 3.1. Let

$$
\begin{aligned}
\hat{\xi}_{i, 1}= & \frac{1}{b_{i, \rho}}\left(-k_{0 i} \epsilon_{i}-\hat{k}_{i} \zeta_{i}+\left(1-\delta_{i}\right) h^{T} \dot{\hat{w}}_{0 i}\right. \\
& \left.-h^{T} \hat{w}_{i, 1}-h^{T} \hat{w}_{i, 2}\right)
\end{aligned}
$$

where $\hat{w}_{i, 1}$ and $\hat{w}_{i, 2}$ are estimates of $w_{i, 1}$ and $w_{i, 2}$ respectively, and the resultant dynamics of $\epsilon_{i}$ is given by

$$
\begin{aligned}
\dot{\epsilon}_{i}= & \tilde{z}_{i, 1}+\psi_{i, y} e_{i}-k_{0 i} \epsilon_{i}-\hat{k}_{i} \zeta_{i}+h^{T} \tilde{w}_{i, 1}+h^{T} \tilde{w}_{i, 2} \\
& +b_{i, \rho} \tilde{\xi}_{i, 1}
\end{aligned}
$$

where $\tilde{\xi}_{i, 1}=\xi_{i, 1}-\hat{\xi}_{i, 1}, \tilde{w}_{i, 1}=w_{i, 1}-\hat{w}_{i, 1}, \tilde{w}_{i, 2}=w_{i, 2}-\hat{w}_{i, 2}$.

In the second step of the adaptive backstepping, we start with

$$
\dot{\tilde{\xi}}_{i, 1}=-\lambda_{1} \xi_{i, 1}+\xi_{i, 2}-\dot{\hat{\xi}}_{i, 1} .
$$

Note that $\hat{\xi}_{i, 1}$ is a function of $\epsilon_{i}, \zeta_{i}, \hat{k}_{i}, \hat{w}_{0 i}, \hat{w}_{i, 1}$ and $\hat{w}_{i, 2}$. Hence, we have

$$
\begin{aligned}
\dot{\tilde{\xi}}_{i, 1}= & -\lambda_{1} \xi_{i, 1}+\xi_{i, 2}-\frac{\partial \hat{\xi}_{i, 1}}{\partial \hat{k}_{i}} \dot{\hat{k}}_{i}-\frac{\partial \hat{\xi}_{i, 1}}{\partial \hat{w}_{0 i}} \dot{\hat{w}}_{0 i}-\frac{\partial \hat{\xi}_{i, 1}}{\partial \hat{w}_{i, 1}} \dot{\hat{w}}_{i, 1} \\
& -\frac{\partial \hat{\xi}_{i, 1}}{\partial \hat{w}_{i, 2}} \dot{\hat{w}}_{i, 2}-\frac{\partial \hat{\xi}_{i, 1}}{\partial \hat{\epsilon}_{i}}\left(-\left(1-\delta_{i}\right) h^{T} \dot{\hat{w}}_{0 i}+\tilde{z}_{i, 1}\right. \\
& \left.+\psi_{i, y} e_{i}+h^{T} w_{i, 1}+h^{T} w_{i, 2}+b_{i, \rho} \xi_{i, 1}\right) \\
& -\frac{\partial \hat{\xi}_{i, 1}}{\partial \zeta_{i}} \sum_{j=1}^{N} q_{i j}\left(\tilde{z}_{j, 1}+\psi_{j, y} e_{j}\right. \\
& \left.+h^{T} w_{j, 2}+b_{j, \rho} \xi_{j, 1}\right) .
\end{aligned}
$$

Based on (38), we design $\hat{\xi}_{i, 2}$ for $\rho=2$ as

$$
\begin{aligned}
\hat{\xi}_{i, 2}= & \lambda_{1} \xi_{i, 1}-b_{i, \rho} \epsilon_{i}-k_{2,1} \tilde{\xi}_{i, 1}-k_{2,2}\left(\frac{\partial \hat{\xi}_{i, 1}}{\partial \epsilon_{i}}\right)^{2} \tilde{\xi}_{i, 1} \\
& -k_{2,3}\left(\frac{\partial \hat{\xi}_{i, 1}}{\partial \zeta_{i}}\right)^{2} \tilde{\xi}_{i, 1}+\frac{\partial \hat{\xi}_{i, 1}}{\partial \hat{k}_{i}} \dot{\hat{k}}_{i}+\frac{\partial \hat{\xi}_{i, 1}}{\partial \hat{w}_{0 i}} \dot{\hat{w}}_{0 i} \\
& +\frac{\partial \hat{\xi}_{i, 1}}{\partial \hat{w}_{i, 1}} \dot{\hat{w}}_{i, 1}+\frac{\partial \hat{\xi}_{i, 1}}{\partial \hat{w}_{i, 2}} \dot{\hat{w}}_{i, 2}+\frac{\partial \hat{\xi}_{i, 1}}{\partial \hat{\epsilon}_{i}}\left(-\left(1-\delta_{i}\right) h^{T} \dot{\hat{w}}_{0 i}\right. \\
& \left.+h^{T} \hat{w}_{i, 1}+h^{T} \hat{w}_{i, 2}+b_{i, \rho} \xi_{i, 1}\right) \\
& +\frac{\partial \hat{\xi}_{i, 1}}{\partial \zeta_{i}} \sum_{j=1}^{N} q_{i j}\left(h^{T} \hat{w}_{j i, 2}+b_{j, \rho} \xi_{j, 1}\right)
\end{aligned}
$$

where $k_{2,1}, k_{2,2}$ and $k_{2,3}$ are positive constant design parameters, and $\hat{w}_{j i, 2}$ is an estimate of $w_{j, 2}$ that is kept in $i$ th subsystem, and subsequently we denote $\tilde{w}_{j i, 2}=w_{i, 2}-\hat{w}_{j i, 2}$. The resultant dynamics of $\tilde{\xi}_{i, 1}$ are obtained as

$$
\begin{aligned}
\dot{\tilde{\xi}}_{i, 1}= & -b_{i, \rho} \epsilon_{i}-k_{2,1} \tilde{\xi}_{i, 1}+\tilde{\xi}_{i, 2} \\
& -k_{2,2}\left(\frac{\partial \hat{\xi}_{i, 1}}{\partial \epsilon_{i}}\right)^{2} \tilde{\xi}_{i, 1}-k_{2,3}\left(\frac{\partial \hat{\xi}_{i, 1}}{\partial \zeta_{i}}\right)^{2} \tilde{\xi}_{i, 1} \\
& -\frac{\partial \hat{\xi}_{i, 1}}{\partial \hat{\epsilon}_{i}}\left(\tilde{z}_{i, 1}+\psi_{i, y} e_{i}+h^{T} \tilde{w}_{i, 1}+h^{T} \tilde{w}_{i, 2}\right) \\
& -\frac{\partial \hat{\xi}_{i, 1}}{\partial \zeta_{i}} \sum_{j=1}^{N} q_{i j}\left(\tilde{z}_{j, 1}+\psi_{j, y} e_{j}+h^{T} \tilde{w}_{j i, 2}\right) .
\end{aligned}
$$

If $\rho=2$, we have $\tilde{\xi}_{i, 2}=0$ and we can set

$$
u_{i}=\hat{\xi}_{i, 2} .
$$

Then the adaptive laws are given by

$$
\begin{aligned}
\dot{\hat{k}}_{i} & =\epsilon_{i} \zeta_{i}, \\
\dot{\hat{w}}_{0 i} & =S \hat{w}_{0 i}-\left(1-\delta_{i}\right) \zeta_{i} h, \\
\dot{\hat{w}}_{i, 1} & =S \hat{w}_{i, 1}+\epsilon_{i} h-\tilde{\xi}_{i, 1} \frac{\partial \hat{\xi}_{i, 1}}{\partial \hat{\epsilon}_{i}} h, \\
\dot{\hat{w}}_{i, 2} & =S \hat{w}_{i, 2}+\epsilon_{i} h-\tilde{\xi}_{i, 1} \frac{\partial \hat{\xi}_{i, 1}}{\partial \hat{\epsilon}_{i}} h, \\
\dot{\hat{w}}_{j i, 2} & =S \hat{w}_{j i, 2}-\tilde{\xi}_{i, 1}\left(\frac{\partial \hat{\xi}_{i, 1}}{\partial \zeta_{i}}\right) q_{i j} h, j=1, \ldots, N .
\end{aligned}
$$

Note that the control inputs shown in (41) and the adaptive laws shown above are still distributed. This means the control and adaptive laws of the $i$ th subsystem use the information of the subsystems that are connected the $i$ th subsystem.

From the design procedures shown for $\rho=2$, it is expected that control design can be carried out for $\rho>2$ using adaptive backstepping, but more tedious. We show the stability analysis for the case of $\rho=2$ first.

Theorem 5.1 For the network connected heterogeneous unknown linear subsystems (1) with relative degree $\rho=2$, the distributed 
control input specified in (41) and (39) together with the adaptive laws (42), (43), (44), (45) and (46) solve the consensus output tracking problem under Assumptions 1, 2, 3and 4, in the sense that the regulation error $e_{i}$ converges to zero asymptotically for $i=1, \ldots, N$. Proof. Let

$$
\begin{aligned}
V_{\epsilon 2}= & \frac{1}{2} \sum_{i=1}^{N} g_{i}\left(\epsilon_{i}^{2}+\tilde{\xi}_{i, 1}^{2}+k \tilde{w}_{0 i}^{T} \tilde{w}_{0 i}+\tilde{w}_{i, 1}^{T} \tilde{w}_{i, 1}\right. \\
& \left.+\tilde{w}_{i, 2}^{T} \tilde{w}_{i, 2}+\sum_{j=1}^{N} \tilde{w}_{j i, 2}^{T} \tilde{w}_{j i, 2}+\tilde{k}_{i}^{2}\right) .
\end{aligned}
$$

By (36), (40), and the adaptive laws (44), (45) and (46), we have

$$
\begin{aligned}
\dot{V}_{\epsilon 2}= & \sum_{i=1}^{N} g_{i}\left(-k_{0 i} \epsilon_{i}^{2}-\hat{k}_{i} \epsilon_{i} \zeta_{i}+\epsilon_{i} \tilde{z}_{i, 1}+\epsilon_{i} \psi_{i, y} e_{i}\right. \\
& +k \tilde{w}_{0 i}^{T} \dot{\tilde{w}}_{0 i}-\left(k-\hat{k}_{i}\right) \dot{\hat{k}}_{i}-k_{2,1} \tilde{\xi}_{i, 1}^{2}-k_{2,2}\left(\frac{\partial \hat{\xi}_{i, 1}}{\partial \epsilon_{i}}\right)^{2} \tilde{\xi}_{i, 1}^{2} \\
& -k_{2,3}\left(\frac{\partial \hat{\xi}_{i, 1}}{\partial \zeta_{i}}\right)^{2} \tilde{\xi}_{i, 1}^{2}-\frac{\partial \hat{\xi}_{i, 1}}{\partial \epsilon_{i}}\left(\tilde{\xi}_{i, 1} \tilde{z}_{i, 1}+\tilde{\xi}_{i, 1} \psi_{i, y} e_{i}\right) \\
& \left.-\frac{\partial \hat{\xi}_{i, 1}}{\partial \zeta_{i}} \sum_{j=1}^{N} q_{i j}\left(\tilde{\xi}_{i, 1} \tilde{z}_{j, 1}+\tilde{\xi}_{i, 1} \psi_{j, y} e_{j}\right)\right)
\end{aligned}
$$

where we have used the property that $S$ is skew-symmetric. Following the same procedure in the proof of Theorem 4.1 in dealing with the term $k \epsilon_{i} \zeta_{i}$, and similar procedures in dealing with other cross terms, we can obtain that

$$
\begin{aligned}
\dot{V}_{\epsilon 2} \leq & -\frac{1}{2} k r_{0}\|e\|^{2}+\sum_{i=1}^{N} g_{i}\left(-\left(k_{0 i}-2 \kappa_{1}\right) \epsilon_{i}^{2}+\frac{1}{4 \kappa_{1}}\left\|\tilde{z}_{i}\right\|^{2}\right. \\
& +\frac{\psi_{i, y}^{2}}{4 \kappa_{1}} e_{i}^{2}-k_{2,1} \tilde{\xi}_{i, 1}^{2}-\left(k_{2,2}-2 \kappa_{2}\right)\left(\frac{\partial \hat{\xi}_{i, 1}}{\partial \epsilon_{i}}\right)^{2} \tilde{\xi}_{i, 1}^{2} \\
& -\left(k_{2,3}-2 N \kappa_{3}\right)\left(\frac{\partial \hat{\xi}_{i, 1}}{\partial \zeta_{i}}\right)^{2} \tilde{\xi}_{i, 1}^{2}+\frac{1}{4 \kappa_{2}}\left(\tilde{z}_{i, 1}^{2}+\psi_{i, y}^{2} e_{i}^{2}\right) \\
& \left.+\frac{1}{4 \kappa_{3}} \sum_{j=1}^{N} q_{i j}^{2}\left(\tilde{z}_{j, 1}^{2}+\psi_{j, y}^{2} e_{j}^{2}\right)\right) \\
\leq & -\left(\frac{1}{2} k r_{0}-\frac{\bar{g} \bar{\psi}}{4}\left(\frac{1}{\kappa_{1}}+\frac{1}{\kappa_{2}}+\frac{N \bar{q}}{\kappa_{3}}\right)\right)\|e\|^{2} \\
& +\frac{\bar{g}}{4}\left(\frac{1}{\kappa_{1}}+\frac{1}{\kappa_{2}}+\frac{N \bar{q}}{\kappa_{3}}\right) \sum_{i=1}^{N}\left\|\tilde{z}_{i}\right\|^{2}
\end{aligned}
$$

where $\kappa_{i}$ for $i=1,2,3$ are positive constants with $\kappa_{1}<$ $\frac{1}{2} \min _{i}\left\{k_{0 i}\right\}, \kappa_{2}<\frac{1}{2} k_{2,2}, \kappa_{3}<\frac{1}{2 N} k_{2,3}$, and $\bar{g}:=\max _{i}\left\{g_{i}\right\}$, and $\bar{\psi}:=\max _{i}\left\{\psi_{i, y}^{2}\right\}$ and $\bar{q}:=\max _{i}\left\{q_{i i}^{2}\right\}$.

As the stability analysis of $\tilde{z}_{i}$ has been carried out in the previous section through the Lyapunov function $V_{z}$ in (31), we define

$$
V_{2}=V_{\epsilon 2}+\frac{\bar{g}}{4}\left(\frac{1}{\kappa_{1}}+\frac{1}{\kappa_{2}}+\frac{N \bar{q}}{\kappa_{3}}\right) V_{z}
$$

From (49) and (32), we have

$$
\begin{aligned}
\dot{V}_{2} \leq & -\left(\frac{1}{2} k r_{0}-\frac{\bar{g}\left(\bar{\psi}+\bar{p}_{d}\right)}{4}\left(\frac{1}{\kappa_{1}}+\frac{1}{\kappa_{2}}+\frac{N \bar{q}}{\kappa_{3}}\right)\right)\|e\|^{2} \\
& -\frac{\bar{g}}{4}\left(\frac{1}{\kappa_{1}}+\frac{1}{\kappa_{2}}+\frac{N \bar{q}}{\kappa_{3}}\right) \sum_{i=1}^{N}\left\|\tilde{z}_{i}\right\|^{2} \\
= & -\kappa_{02}\|e\|^{2}-\frac{\bar{g}}{4}\left(\frac{1}{\kappa_{1}}+\frac{1}{\kappa_{2}}+\frac{N \bar{q}}{\kappa_{3}}\right) \sum_{i=1}^{N}\left\|\tilde{z}_{i}\right\|^{2}
\end{aligned}
$$

by setting $k=\frac{2}{r_{0}}\left(\kappa_{02}-\frac{\bar{g}\left(\bar{\psi}+\bar{p}_{d}\right)}{4}\left(\frac{1}{\kappa_{1}}+\frac{1}{\kappa_{2}}+\frac{N \bar{q}}{\kappa_{3}}\right)\right)$ with $\kappa_{02}>0$. Hence, we have the boundedness of all the variables, $e \in L_{2} \cap L_{\infty}$, $\tilde{z}_{i} \in L_{2} \cap L_{\infty}$, for $i=1, \ldots, N$, and furthermore from Babalat's lemma, and that $\lim _{t \rightarrow \infty} e(t)=0$ and $\lim _{t \rightarrow \infty} \tilde{z}_{i}(t)=0$, for $i=$ $1, \ldots, N$.

For the case of $\rho>2$, the backstepping procedures can be carried on in a similar way to the case of $\rho=2$, but, clearly more variables will be involved in the design, which is a nature of backstepping design, especially in the case of backstepping of network-connected systems. It is important to clearly check the possible propagation of design variables needed in the network communication through the backsetpping design. In the design shown here, the variables in other subsystems will be involved through the variable $\zeta_{i}$ in the $i$ th subsystem. As shown in the control design for the case of $\rho=2$, through the derivatives of $\zeta_{i}$, the other variables, $w_{j, 2}$ and $\xi_{j, 1}$ are also involved. As a local copy, $\hat{w}_{j i, 2}$, is used for the estimation of $w_{j, 2}$ in the $i$ th subsystem, its derivative remains local. In the next step, for $\rho=3$, their derivatives will appear in the control design. It is clear that the derivative of $\xi_{j, 1}$ does not involved the interaction with other subsystems. The derivative of $\xi_{j, 1}$ does not propagate to other subsystems. In the subsequent steps, for the control design of the $i$ th subsystem, the involvements will be limited to the variables $\hat{w}_{j i, 2}$, and $\xi_{j, r}, r=1, \ldots, \rho-2$, if $q_{i j} \neq 0$, in addition to $\zeta_{i}$. Hence, we can conclude that the variables used for the control design are the variables from the neighbouring subsystems, and no further propagation in the network connection to other subsystems, because of the specific control design with the introduction of the estimated unknown trajectory. For the convenience of of the subsequent presentation, we define $\mathcal{N}_{i}:=\left\{j \mid q_{i j} \neq 0\right\}$, i.e., $\mathcal{N}_{i}$ is the set that contains the index of the subsystems in the neighbourhood of the $i$ th subsystem. We also introduce a generic index $\bar{j}_{i}$ to represent any element in $\mathcal{N}_{i}$. For example, $\xi_{\bar{j}_{i}, 1}$ represents $\xi_{j, 1} \mathrm{~s}$ for $j \in \mathcal{N}_{i}$.

For $\rho>2$, we need to use backsteping for $r$ steps with $r=\rho$. The steps for $r=1,2$ have been shown, and the following step shows the design for $r>2$. Note that the tuning functions are normally used in adaptive backstepping when $\rho>2$ to avoid multiple copies of estimated parameters in backstepping. To carry on the adaptive backstepping, we need to replace $\dot{\hat{w}}_{i, 1} \dot{\hat{w}}_{i, 2}$ in $\hat{\xi}_{i, 2}$ (39) by $\tau_{i 1, r}$ and $\tau_{i 2, r}$, the corresponding tuning functions for $\hat{w}_{i, 1}$ and $\hat{w}_{i, 2}$.

In the $r$ th step of the adaptive backstepping, $r=3, \ldots, \rho$, we start with

$$
\dot{\tilde{\xi}}_{i, r-1}=-\lambda_{r-1} \xi_{i, r-1}+\xi_{i, r}-\dot{\hat{\xi}}_{i, r-1} .
$$

Based on the previous discussion, we know that $\hat{\xi}_{i, r-1}$ is a function of $\epsilon_{i}, \zeta_{i}, \hat{k}_{i}, \hat{w}_{0 i}, \hat{w}_{i, 1}, \hat{w}_{i, 2}, \hat{w}_{\bar{j}_{i} i, 2}$ and $\xi_{\bar{j}_{i}, m}$ for $m=1, \ldots, r-2$. Hence, we have

$$
\begin{aligned}
\dot{\tilde{\xi}}_{i, r-1}= & -\lambda_{r-1} \xi_{i, r-1}+\xi_{i, r}-\frac{\partial \hat{\xi}_{i, r-1}}{\partial \hat{k}_{i}} \dot{\hat{k}}_{i} \\
& -\frac{\partial \hat{\xi}_{i, r-1}}{\partial \hat{w}_{0 i}} \dot{\hat{w}}_{0 i}-\frac{\partial \hat{\xi}_{i, r-1}}{\partial \hat{w}_{i, 1}} \dot{\hat{w}}_{i, 1}-\frac{\partial \hat{\xi}_{i, r-1}}{\partial \hat{w}_{i, 2}} \dot{\hat{w}}_{i, 2} \\
& -\frac{\partial \hat{\xi}_{i, r-1}}{\partial \hat{\epsilon}_{i}}\left(-\left(1-\delta_{i}\right) h^{T} \dot{\hat{w}}_{0 i}+\tilde{z}_{i, 1}+\psi_{i, y} e_{i}\right. \\
& \left.+h^{T} w_{i, 1}+h^{T} w_{i, 2}+b_{i, \rho} \xi_{i, 1}\right) \\
& -\frac{\partial \hat{\xi}_{i, r-1}}{\partial \zeta_{i}} \sum_{j=1}^{N} q_{i j}\left(\tilde{z}_{j, 1}+\psi_{j, y} e_{j}+h^{T} w_{j, 2}\right. \\
& \left.+b_{i, \rho} \xi_{j, 1}\right)-\sum_{j \in \mathcal{N}_{i}} \frac{\partial \hat{\xi}_{i, r-1}}{\partial \hat{w}_{j i, 2}} \dot{\hat{w}}_{j i, 2} \\
& -\sum_{j \in \mathcal{N}_{i}} \sum_{m=1}^{r-2} \frac{\partial \hat{\xi}_{i, r-1}}{\partial \xi_{j, m}}\left(-\lambda_{m} \xi_{j, m}+\xi_{j, m+1}\right) .
\end{aligned}
$$


Based on (51), we design $\hat{\xi}_{i, r}$ as

$$
\begin{aligned}
\hat{\xi}_{i, r}= & \lambda_{r-1} \xi_{i, r-1}-\tilde{\xi}_{r-2}-k_{r, 1} \tilde{\xi}_{i, r-1} \\
& -k_{r, 2}\left(\frac{\partial \hat{\xi}_{i, r-1}}{\partial \epsilon_{i}}\right)^{2} \tilde{\xi}_{i, r-1}-k_{r, 3}\left(\frac{\partial \hat{\xi}_{i, r-1}}{\partial \zeta_{i}}\right)^{2} \tilde{\xi}_{i, r-1} \\
& +\frac{\partial \hat{\xi}_{i, r-1}}{\partial \hat{k}_{i}} \dot{\hat{k}}_{i}+\frac{\partial \hat{\xi}_{i, r-1}}{\partial \hat{w}_{0 i}} \dot{\hat{w}}_{0 i}+\frac{\partial \hat{\xi}_{i, r-1}}{\partial \hat{w}_{i, 1}} \tau_{i 1, r} \\
& +\beta_{i 1, r}+\frac{\partial \hat{\xi}_{i, r-1}}{\partial \hat{w}_{i, 2}} \tau_{i 2, r}+\beta_{i 2, r} \\
& +\frac{\partial \hat{\xi}_{i, r-1}}{\partial \hat{\epsilon}_{i}}\left(-\left(1-\delta_{i}\right) h^{T} \dot{\hat{w}}_{0 i}+h^{T} \hat{w}_{i, 1}\right. \\
& +\frac{\partial \hat{\xi}_{i, r-1}}{\partial \zeta_{i}} \sum_{j=1}^{N} q_{i j}\left(h^{T} \hat{w}_{j i, 2}+b_{j, \rho} \xi_{j, 1}\right) \\
& +\sum_{j \in \mathcal{N}_{i}} \frac{\partial \hat{\xi}_{i, r-1}}{\partial \hat{w}_{j i, 2}} \tau_{j i 2, r}+\sum_{j \in \mathcal{N}_{i}} \beta_{j i 2, r} \\
& +\sum_{j \in \mathcal{N}_{i}} \sum_{m=1}^{r-2} \frac{\partial \hat{\xi}_{i, r-1}}{\partial \xi_{j, m}}\left(-\lambda_{m} \xi_{j, m}+\xi_{j, m+1}\right)
\end{aligned}
$$

where $k_{r, 1}, k_{r, 2}$ and $k_{r, 3}$ are positive constant design parameters; $\tau_{j i 2, r}$ is the tuning function for $\hat{w}_{j i, 2}$; and $\beta_{i 1, r}, \beta_{i 2, r}$ and $\beta_{j i 2, r}$ the complementing functions for the tuning functions [18]. The resultant dynamics of $\tilde{\xi}_{i, r}$ are obtained as

$$
\begin{aligned}
\dot{\tilde{\xi}}_{i, r-1}= & -\tilde{\xi}_{r-2}+\tilde{\xi}_{i, r}-k_{r, 1} \tilde{\xi}_{i, r-1} \\
& -k_{r, 2}\left(\frac{\partial \hat{\xi}_{i, r-1}}{\partial \epsilon_{i}}\right)^{2} \tilde{\xi}_{i, r-1}-k_{r, 3}\left(\frac{\partial \hat{\xi}_{i, r-1}}{\partial \zeta_{i}}\right)^{2} \tilde{\xi}_{i, r-1} \\
& -\frac{\partial \hat{\xi}_{i, r-1}}{\partial \hat{w}_{i, 1}}\left(\dot{\hat{w}}_{i, 1}-\tau_{i 1, r}\right)+\beta_{i 1, r} \\
& -\frac{\partial \hat{\xi}_{i, r-1}}{\partial \hat{w}_{i, 2}}\left(\dot{\hat{w}}_{i, 2}-\tau_{i 2, r}\right)+\beta_{i 2, r} \\
& -\frac{\partial \hat{\xi}_{i, r-1}}{\partial \hat{\epsilon}_{i}}\left(\tilde{z}_{i, 1}+\psi_{i, y} e_{i}+h^{T} \tilde{w}_{i, 1}+h^{T} \tilde{w}_{i, 2}\right) \\
& -\frac{\partial \hat{\xi}_{i, r-1}}{\partial \zeta_{i}} \sum_{j=1}^{N} q_{i j}\left(\tilde{z}_{j, 1}+\psi_{j, y} e_{j}+h^{T} \tilde{w}_{j i, 2}\right) . \\
& -\sum_{j \in \mathcal{N}_{i}} \frac{\partial \hat{\xi}_{i, r-1}}{\partial \hat{w}_{j i, 2}}\left(\dot{\hat{w}}_{j i, 2}-\tau_{j i 2, r}\right)+\sum_{j \in \mathcal{N}_{i}} \beta_{j i 2, r}
\end{aligned}
$$

The adaptive laws for $\hat{k}_{i}$ and $\hat{w}_{0 i}$ remain the same as for $\rho=2$ and the tuning functions are designed as

$$
\begin{aligned}
& \tau_{i 1, r}=S \hat{w}_{i, 1}+\epsilon_{i} h-\sum_{m=1}^{r-1} \tilde{\xi}_{i, m} \frac{\partial \hat{\xi}_{i, m}}{\partial \hat{\epsilon}_{i}} h, \\
& \tau_{i 2, r}=S \hat{w}_{i, 2}+\epsilon_{i} h-\sum_{m=1}^{r-1} \tilde{\xi}_{i, m} \frac{\partial \hat{\xi}_{i, m}}{\partial \hat{\epsilon}_{i}} h, \\
& \tau_{j i 2, r}=S \hat{w}_{j i, 2}-\sum_{m=2}^{r-1} \tilde{\xi}_{i, m} \frac{\partial \hat{\xi}_{i, m}}{\partial \zeta_{i}} q_{i j} h \\
& j=1, \ldots, N .
\end{aligned}
$$

and we set $\tau_{j i 2, r}=0$, for $r=1,2$. The complementing functions are designed as

$$
\begin{aligned}
& \beta_{i 1, r}=\sum_{m=2}^{r-1} \tilde{\xi}_{i, m} \frac{\partial \hat{\xi}_{i, m}}{\hat{w}_{i, 1}} \frac{\partial \hat{\xi}_{i, m}}{\partial \hat{\epsilon}_{i}} h, \\
& \beta_{i 2, r}=\sum_{m=2}^{r-1} \tilde{\xi}_{i, m} \frac{\partial \hat{\xi}_{i, m}}{\hat{w}_{i, 2}} \frac{\partial \hat{\xi}_{i, m}}{\partial \hat{\epsilon}_{i}} h,
\end{aligned}
$$

$$
\begin{gathered}
\beta_{j i 2, r}=\sum_{m=3}^{r-1} \tilde{\xi}_{i, m} \frac{\partial \hat{\xi}_{i, m}}{\hat{w}_{j i, 2}} \frac{\partial \hat{\xi}_{i, m}}{\partial \zeta_{i}} q_{i j} h \\
j=1, \ldots, N,
\end{gathered}
$$

and we set $\beta_{i 1, r}=0, \beta_{i 2, r}=0$ for $r=1,2$, and $\beta_{j i 2, r}=0$ for $r=1,2,3$.

If the relative degree $\rho=r$, we have $\tilde{\xi}_{i, r}=0$ and we can set

$$
u_{i}=\hat{\xi}_{i, r}
$$

and the other adaptive laws as

$$
\begin{aligned}
\dot{\hat{w}}_{i, 1} & =\tau_{i 1, \rho}, \\
\dot{\hat{w}}_{i, 2} & =\tau_{i 2, \rho}, \\
\dot{\hat{w}}_{j i, 2} & =\tau_{j i 2, \rho}, \quad j=1, \ldots, N .
\end{aligned}
$$

The complete stability analysis can be carried out in a similar way to that for $\rho=2$, but with much more extra terms. Nevertheless, it is worth showing the details to clarify the proposed control design. Theorem 6.1 For the network connected heterogeneous unknown linear subsystems (1) with relative degree $\rho>2$, the distributed control input specified in (60) and (52) together with the adaptive laws (42), (43), (61), (62) and (63) solve the consensus output tracking problem under Assumptions 1, 2, 3 and 4, in the sense that the regulation error $e_{i}$ converges to zero asymptotically for $i=1, \ldots, N$. Proof. Let

$$
\begin{aligned}
V_{\epsilon \rho}= & \frac{1}{2} \sum_{i=1}^{N} g_{i}\left(\epsilon_{i}^{2}+\sum_{m=1}^{\rho-1} \tilde{\xi}_{m, 1}^{2}+k \tilde{w}_{0 i}^{T} \tilde{w}_{0 i}+\tilde{w}_{i, 1}^{T} \tilde{w}_{i, 1}\right. \\
& \left.+\tilde{w}_{i, 2}^{T} \tilde{w}_{i, 2}+\sum_{j \in \mathcal{N}_{i}} \tilde{w}_{j i, 2}^{T} \tilde{w}_{j i, 2}+\tilde{k}_{i}^{2}\right) .
\end{aligned}
$$

By (36), (53), and the adaptive laws (42), (43), (61), (62) and (63), we have

$$
\begin{aligned}
\dot{V}_{\epsilon \rho}= & \sum_{i=1}^{N} g_{i}\left(-k_{0 i} \epsilon_{i}^{2}-\hat{k}_{i} \epsilon_{i} \zeta_{i}+\epsilon_{i} \tilde{z}_{i, 1}+\epsilon_{i} \psi_{i, y} e_{i}\right. \\
& +k \tilde{w}_{0 i}^{T} \dot{\tilde{w}}_{0 i}-\left(k-\hat{k}_{i}\right) \dot{\hat{k}}_{i} \\
& +\sum_{m=1}^{\rho-1}\left(-k_{m+1,1} \tilde{\xi}_{i, m}^{2}-k_{m+1,2}\left(\frac{\partial \hat{\xi}_{i, m}}{\partial \epsilon_{i}}\right)^{2} \tilde{\xi}_{i, m}^{2}\right. \\
& -k_{m+1,3}\left(\frac{\partial \hat{\xi}_{i, m}}{\partial \zeta_{i}}\right)^{2} \tilde{\xi}_{i, m}^{2}-\frac{\partial \hat{\xi}_{i, m}}{\partial \hat{\epsilon}_{i}}\left(\tilde{\xi}_{i, m} \tilde{z}_{i, 1}+\tilde{\xi}_{i, m} \psi_{i, y} e_{i}\right) \\
& \left.\left.-\frac{\partial \hat{\xi}_{i, m}}{\partial \zeta_{i}} \sum_{j=1}^{N} q_{i j}\left(\tilde{\xi}_{i, m} \tilde{z}_{j, 1}+\tilde{\xi}_{i, m} \psi_{j, y} e_{j}\right)\right)\right)
\end{aligned}
$$

where the skew-symmetric property of $S$ is used again. Following the same procedure in the proof of the previous theorems in dealing with the term $k \epsilon_{i} \zeta_{i}$, and similar procedures in dealing with other cross terms, we can obtain that

$$
\begin{aligned}
\dot{V}_{\epsilon \rho} \leq & -\frac{1}{2} k r_{0}\|e\|^{2}+\sum_{i=1}^{N} g_{i}\left(-\left(k_{0 i}-2 \kappa_{1}\right) \epsilon_{i}^{2}+\frac{1}{4 \kappa_{1}}\left\|\tilde{z}_{i}\right\|^{2}\right. \\
& +\frac{\psi_{i, y}^{2}}{4 \kappa_{1}} e_{i}^{2}+\sum_{m=1}^{\rho-1}\left(-k_{m+1,1} \tilde{\xi}_{i, m}^{2}\right. \\
& -\left(k_{m+1,2}-2 \kappa_{2}\right)\left(\frac{\partial \hat{\xi}_{i, m}}{\partial \epsilon_{i}}\right)^{2} \tilde{\xi}_{i, m}^{2} \\
& -\left(k_{m+1,3}-2 N \kappa_{3}\right)\left(\frac{\partial \hat{\xi}_{i, m}}{\partial \zeta_{i}}\right)^{2} \tilde{\xi}_{i, m}^{2} \\
& \left.\left.+\frac{1}{4 \kappa_{2}}\left(\tilde{z}_{i, 1}^{2}+\psi_{i, y}^{2} e_{i}^{2}\right)+\frac{1}{4 \kappa_{3}} \sum_{j=1}^{N} q_{i j}^{2}\left(\tilde{z}_{j, 1}^{2}+\psi_{j, y}^{2} e_{j}^{2}\right)\right)\right)
\end{aligned}
$$




$$
\leq-\left(\frac{1}{2} k r_{0}-\frac{\bar{g} \bar{\psi}}{4 \kappa}\right)\|e\|^{2}+\frac{\bar{g}}{4 \kappa} \sum_{i=1}^{N}\left\|\tilde{z}_{i}\right\|^{2}
$$

where $\kappa_{i}$ for $i=1,2,3$ are positive constants with the refined conditions $\kappa_{1}<\frac{1}{2} \min _{i}\left\{k_{0 i}\right\}, \kappa_{2}<\frac{1}{2} \min _{r}\left\{k_{r, 2}\right\}, \kappa_{3}<\frac{1}{2 N} \min _{r}\left\{k_{r, 3}\right\}$, $\frac{1}{\kappa}=\frac{1}{\kappa_{1}}+\frac{\rho-1}{\kappa_{2}}+\frac{(\rho-1) N \bar{q}}{\kappa_{3}}$, and the other notations remain the same, i.e., $\bar{g}:=\max _{i}\left\{g_{i}\right\}$, and $\bar{\psi}:=\max _{i}\left\{\psi_{i, y}^{2}\right\}$ and $\bar{q}:=\max _{i}\left\{q_{i i}^{2}\right\}$.

Again, using the stability property of $\tilde{z}_{i}$ established through the Lyapunov function $V_{z}$ in (31), we define

$$
V_{\rho}=V_{\epsilon \rho}+\frac{\bar{g}}{4 \kappa} V_{z}
$$

From (66) and (32), we have

$$
\begin{aligned}
\dot{V}_{\rho} & \leq-\left(\frac{1}{2} k r_{0}-\frac{\bar{g}\left(\bar{\psi}+\bar{p}_{d}\right)}{4 \kappa}\right)\|e\|^{2}-\frac{\bar{g}}{4 \kappa} \sum_{i=1}^{N}\left\|\tilde{z}_{i}\right\|^{2} \\
& =-\kappa_{02}\|e\|^{2}-\frac{\bar{g}}{4 \kappa} \sum_{i=1}^{N}\left\|\tilde{z}_{i}\right\|^{2}
\end{aligned}
$$

by setting $k=\frac{2}{r_{0}}\left(\kappa_{02}-\frac{\bar{g}\left(\bar{\psi}+\bar{p}_{d}\right)}{4 \kappa}\right)$ with $\kappa_{02}>0$. Then following the same procedure as that in the proof of Theorem 5.1, we have $\lim _{t \rightarrow \infty} e(t)=0$ and $\lim _{t \rightarrow \infty} \tilde{z}_{i}(t)=0$, for $i=1, \ldots, N$.

\section{EXAMPLE}

An example is used to demonstrate the control design for adaptive consensus output regulation. Consider a network-connected system that consists of 5 subsystems in the same form as (1), of which, for $i=1,3,5$, each subsystem is a second-order linear model, with $\phi_{i}=[1,1,1]^{T}$, and $b_{i}=[2,10]^{T}$, and for $i=2$, 4, each subsystem is a third-order linear model, with $\phi_{i}=[1,1,1]^{T}$ and $b_{i}=[1,8,15]^{T}$. Note that the values of $\phi_{i}$ and $b_{i}$ are not available to the control design. The exosystem matrix is $S=\left[\begin{array}{cc}0 & 1 / 4 \\ -1 / 4 & 0\end{array}\right]$, and the desired system output is only available to subsystem 4 . The adjacency matrix $A$ and the resultant $Q$ are given by

$A=\left[\begin{array}{lllll}0 & 0 & 0 & 1 & 1 \\ 1 & 0 & 0 & 0 & 0 \\ 0 & 1 & 0 & 0 & 0 \\ 0 & 0 & 1 & 0 & 0 \\ 0 & 0 & 0 & 1 & 0\end{array}\right], Q=\left[\begin{array}{ccccc}2 & 0 & 0 & -1 & -1 \\ -1 & 1 & 0 & 0 & 0 \\ 0 & -1 & 1 & 0 & 0 \\ 0 & 0 & -1 & 2 & 0 \\ 0 & 0 & 0 & -1 & 1\end{array}\right]$

The control design follows the procedures for $\rho=1$, and in the simulation study, $k_{0 i}=2.5$. The subsystem outputs under the proposed control are shown in Figure 1.

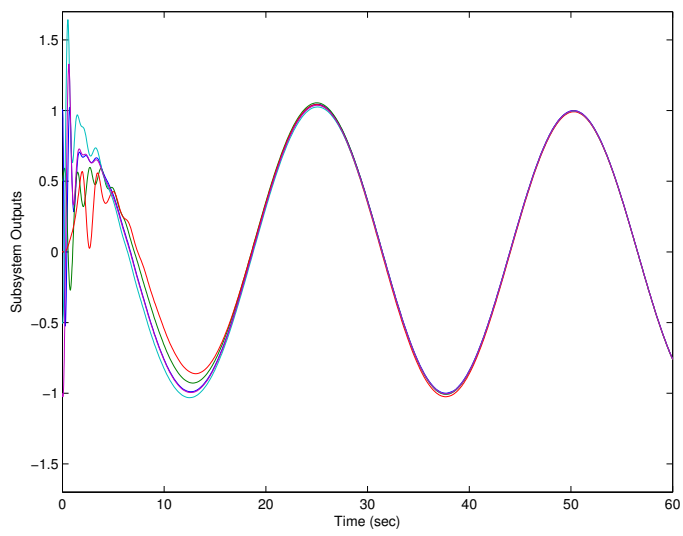

Fig. 1. The subsystem outputs.

\section{CONCLUSION}

We have proposed an adaptive control design for output regulation of network-connected heterogeneous unknown linear systems. The control design successfully explores the structure of the exosystem for the estimation of the regulation errors of the subsystems and the desired feedforward control inputs. The adaptive laws are based on the estimated regulation errors and the control design is fully distributed and ensures the asymptotic convergence of the regulation errors of all the subsystems.

\section{REFERENCES}

[1] R.Olfati-Saber and R. M. Murray, "Consensus problems in networks of agents with switching topology and time-delay," IEEE Trans. Automa. Contr., vol. 49, no. 9, pp. 1520-1533, 2004.

[2] Y. Hong, J. Hu, and L. Gao, "Tracking control for multi-agent consensus with an active leader and variable topology," Automatica, vol. 42, no. 7, pp. 1177-1182, 2006

[3] R. Olfati-Saber, J. A. Fax, and R. M. Murray, "Consensus and cooperation in networked multi-agent systems," Proceedings of the IEEE, vol. 95, no. 1, pp. 215-233, 2007.

[4] Z. Li, Z. Duan, G. Chen, and L. Huang, "Consensus of multiagent systems and synchronization of complex networks, a unified viewpoint," IEEE Trans. Circuits Syst. I., vol. 57, no. 1, pp. 213-224, 2010.

[5] Z. Ding, "Consensus output regulation of a class of heterogeneous nonlinear systems," IEEE Trans. Automatic Control, vol. 58, no. 10, pp. 2648-2653, 2013.

[6] W. Chen, X. Li, W. Ren, and C. Wen, "Adaptive consensus of multiagent systems with unknown identical control directions based on a novel nussbaum-type function," IEEE Trans. Automa. Contr., vol. 59, no. 7, pp. 1887-1892, 2014.

[7] M.-C. Fan, Z. Chen, and H.-T. Zhang, "Semi-global consensus of nonlinear second-order multi-agent systems with measurement output feedback," IEEE Trans. Automa. Contr., vol. 59, no. 8, pp. 2222-2227, 2014.

[8] H. Yu and X. Xia, "Adaptive consensus of multi-agents in networks with jointly connected topologies," Automatica, vol. 48, pp. 1783-1790, 2012.

[9] Y. Su and J. Huang, "Cooperative adaptive output regulation for a class of nonlinear uncertain multi-agent systems with unknown leader," Systems \& Control Letters, vol. 62, no. 6, pp. 461-467, 2013.

[10] Z. Ding, "Adaptive consensus output regulation of a class of nonlinear systems with unknown high-frequency gain," Automatica, vol. 51, pp. 348-355, 2015.

[11] A. Das and F. Lewis, "Distributed adaptive control for synchronization of unknown nonlinear networked systems," Automatica, vol. 46, pp. 2014 2021, 2010.

[12] H. Zhang and F. Lewis, "Adaptive cooperative tracking control of highorder nonlinear systems with unknown dynamics," Automatica, vol. 48, pp. 1432-1439, 2012.

[13] W. Wang, J. Huang, C. Wen, and H. Fan, "Distributed adaptive control for consensus tracking with application to formation control of nonholonomic mobile robots," Automatica, vol. 50, pp. 1254-1263, 2014.

[14] Z. Li, G. Wen, Z. Duan, and W. Ren, "Designing fully distributed consensus protocols for linear multi-agent systems with directed graphs," IEEE Trans. Automa. Contr, vol. 60, no. 4, pp. 1152-1157, 2015.

[15] Z. Li and Z. Ding, "Distributed adaptive consensus and output tracking of unknown linear systems on directed graphs," Automatica, vol. 55, pp. $12-18,2015$.

[16] Z. Qu, Cooperative Control of Dynamical Systems. London: SpringerVerlag, 2009.

[17] P. A. Ioannou and J. Sun, Robust Adaptive Control. New Jersey: Prentice Hall, 1996.

[18] Z. Ding, Nonlinear and Adaptive Control Systems. London: IET, 2013. 